\title{
THE CALCULUS OF VARIATIONS*
}

\author{
BY LEONIDA TONELLI
}

1. The Purpose of this Note. It is proposed to explain briefly here the method followed in my Fondamenti $d i$ Calcolo delle Variazionit, of which the first two volumes have already been published. In these the simplest problem of the calculus of variations in the plane and the corresponding isoperimetric problem are considered, for integrands which are functions only of the coordinates and the inclination of the tangent at a point of the curve along which the integrals are taken; the other problems of the calculus of variations will be discussed in later volumes.

2. Reasons for the Development of a new Method in the Calculus of Variations. The inconveniences which present themselves in the classical methods of the calculus of variations are well known. Here I emphasize only the fact that, aside from the difficulties inherent in the verification for a given curve of the well known sufficient conditions for an extremum, there is further a serious difficulty which sometimes arrests at the very beginning the application of the classical results. It is that the theory of differential equations offers but few methods of deciding whether or not there exists an extremal joining two points which are not conveniently near to each other. I propose on that account to find a more direct procedure for the solution of the problems of the calculus of variations, independent of the theory of differential equations. At the same time my researches have two other objectives. The first is that of obtaining general results in the domain of absolute extrema, as contrasted with the classical method which is particularly devoted to relative extrema. In the second place it will be interesting to see if the new methods

* Translated into English and prepared for publication by G. A. Bliss.

† Bologna, Zanichelli; vol. 1, 1922, vol. 2, 1924.

$11^{*}$ 
[March-April,

are applicable to classes of curves which are more general than those which are usually considered in the treatises on the calculus of variations. One is necessarily restricted there to more special classes because the methods which are followed are founded essentially upon the consideration of extremals. The classical problem of Newton, the determination of a solid of revolution of least resistance, is a first example of the necessity of considering more general classes of curves than the ordinary ones.

3. Fundamental Conceptions of the new Method. Having thus specified the scope of the proposed investigation a natural method presents itself, that of the functional calculus according to the ideas of Volterra. The integrals which are considered in the calculus of variations, and which it is desired to make a maximum or minimum, depend for the most part upon curves or surfaces and are real valued functions of such curves or surfaces. These belong therefore in the domain of the functional calculus, and it is evident that the principal developments of the calculus of variations can be obtained by constructing, for its functions of curves and surfaces in the domain of the functional calculus, a theory of extrema corresponding to that which is usually developed for functions of one or more variables in the ordinary treatises on analysis. It is precisely this method which is followed here.

A first difficulty presents itself at once. In the ordinary theory of maxima and minima one starts from a fundamental property of the functions there considered, namely, continuity. But continuity cannot be made the basis of the new theory to be constructed, since the functions of curves and surfaces to be considered are in general discontinuous. For greater precision and in order to remain in the domain of the reasoning of the first two volumes of my Fondamenti, let us limit ourselves here to the functions of curves which are integrals whose integrands depend only upon the coordinates and the inclination of the tangent 
at a variable point of the curve along which the integral is taken. If the property of continuity is required for such an integral, it is found that the only integrals which can be considered are those whose integrands are expressible linearly in terms of the slope of the curve of integration; and in the parametric case, the only integrals with this property are those which depend solely upon the coordinates of the end-points of the curve along which the integral is taken.

It is therefore necessary to discard continuity, and to place at the basis of the new theory a conception which is more general and better adapted to the functions which have to be considered.

The analysis of the concept of continuity, for the functions of numerical variables of the ordinary differential calculus, has already led Baire to decompose this concept into two others of a more elementary sort called lower semi-continuity and upper semi-continuity. The simultaneous possession of both these types of semi-continuity implies precisely ordinary continuity. Baire has also observed that the elementary reasoning which permits one to prove that a continuous function in a bounded closed domain has always a minimum or a maximum, is also sufficient to justify the same conclusion in the case when the function possesses lower or upper semi-continuity, respectively. This observation applied to our functions of curves, permits us to overcome the first serious difficulty and to establish the foundations of the new theory.

If the length of arc is taken as the variable of integration, and if the function to be integrated does not depend upon the derivatives of the coordinates of the points of the curve of integration, that is to say, if it is a function $\varphi(x, y)$ of the coordinates $x$ and $y$, then an elementary geometric argument furnishes at once the result that when $\varphi(x, y)$ is always positive, its integral possesses lower semicontinuity as a function of the curve of integration. This property has already been noted in the memorable Thesis of Lebesgue. But is this semi-continuity a property only 
of the integrals of the particular form here indicated, or does it belong to a class of integrals so large that it can be made the basis of a general method of the calculus of variations? My researches, begun in 1911, have permitted me to answer the latter part of this question affirmatively. Among the integrals in the parametric form all those which Hilbert has called regular possess semi-continuity, as well as those which I have named quasi-regular seminormal, and also those which are simply quasi-regular provided that another condition is added such as, for example, the condition that the function under the integral sign does not change sign. Besides these sufficient conditions for semi-continuity I have investigated also necessary conditions, and conditions which are at the same time necessary and sufficient. Conditions of this last sort for lower semi-continuity are that the integral be quasiregular positive, and that every so-called exceptional point shall be the center of at least one circle such that the value of the integral along an arbitrarily chosen closed arc interior to the circle is always positive or zero. By an exceptional point is meant one at which the well known Weierstrassian function $F_{1}$ is identically zero in the derivatives $x^{\prime}, y^{\prime}$.

For the case of a non-parametric integral a necessary and sufficient condition is simply that the integral be quasi-regular.

The semi-continuity hitherto considered is semi-continuity in the entire field, i. e., at every curve of the field. I have also studied semi-continuity at a particular curve and have arrived here at the conclusion that the property of semicontinuity corresponds to the well known condition of Weierstrass in the calculus of variations, a condition which, as is known, implies also that of Legendre. This conclusion seems to me interesting because it permits one to establish a complete correspondence between the conditions for a relative extremum in the differential calculus and those for the same sort of an extremum in the calculus of 
variations. In the differential calculus, for a function $f(x)$ which is continuous and has derivatives of the first two orders, a point $x_{0}$ in the domain where $f(x)$ is defined furnishes a relative minimum for $f(x)$ if at $x_{0}$ the first derivative of the function is zero and the second derivative positive. In the calculus of variations a curve $C_{0}$, interior to the field in which the integral to be minimized is considered, furnishes a relative minimum for the integral if the first variation is zero and the second positive, and if furthermore the conditions of Weierstrass and Legendre are satisfied. But these conditions of Weierstrass and Legendre have precisely the effect of assuring us that the integral in question has lower semi-continuity at the curve $C_{0}$, a condition which in the case of the functions $f(x)$ is already implicitly contained in the hypothesis of continuity. Consequently to the conditions of the differential calculus relative to the vanishing of the first derivative and the positive sign of the second, correspond exactly in the calculus of variations, and with the same significance, the analogous conditions on the first and second variations. And the correspondence in the two fields between the conditions sufficient for a minimum is also repeated in the case of necessary conditions.

The study thus briefly summarized and the observation, which it may be well to repeat explicitly, that every integral must possess lower semi-continuity on each of its minimizing curves, have led me to construct a new development of the calculus of variations founded essentially on the concept of semi-continuity.

4. Existence Theorems for Extrema. A first application of semi-continuity can be made immediately to the demonstration of theorems concerning the existence of an absolute extremum, by following the same method of procedure as that of the differential calculus for extrema of ordinary functions of real variables.

Nevertheless a difficulty presents itself here which has 
[March-April,

no analogue in the theory of extrema of the differential calculus. To establish the existence of an absolute minimum on a given interval for a function $f(x)$ which possesses lower semi-continuity, one uses, as is well known, a minimizing sequence $x_{1}, x_{2}, \cdots, x_{n}, \cdots$; such a sequence has at least one limit point by the theoren of Bolzano-Weirstrass; and in each one of these limit points the function $f(x)$, in virtue of its lower semi-continuity, assumes its minimum value. In the case of the calculus of variations the analogous minimizing sequence of curves $C_{1}, C_{2}, \ldots, C_{n}, \ldots$ does not always have a limiting curve because for classes of curves the theorem of Bolzano-Weierstrass is not always true. One has here the new circumstance that even though an integral possesses lower semi-continuity in a limited domain it may yet have no minimizing curve. To be able to reach a conclusion like that for the case of a function $f(x)$ it is necessary therefore to introduce some new condition which will assure the existence of a limiting element for the minimizing sequence. This further condition may assume various forms from which result numerous theorems of existence for extrema.

For integrals in parametric form I have begun, in the second volume of my Fondamenti, by demonstrating the existence of an extremum for the so-called quasi-regular definite integrals, a special case being the integral of a function $\varphi(x, y)$ which has always the same sign, a case which as I have already remarked was treated by Lebesgue in his Thesis. From the quasi-regular definite integrals I then pass to more general cases which require more profound analysis, seeking always to make the theory inclusive of the more important applications of the calculus of variations.

For the integrals in ordinary form the condition which assures the existence of a limiting element for a minimizing sequence takes a form different from that assumed in the parametric case, in that it is related to the behavior of the integrand function $f\left(x, y, y^{\prime}\right)$ as $y^{\prime}$ tends to infinity. Furthermore a variety of existence theorems are here stated 
designed to satisfy as far as possible the exigencies of the applications, and results are found, as particular cases, which were established in other ways by Hadamard.

The theorems of existence, both for the parametric and ordinary integrals, are stated so as to secure the maximum generality possible with regard to the character of the curves themselves and the character of the class of curves which is to be considered. In the first place the curves are supposed only to be rectifiable; and in the second place the functions $y(x)$ representing them are supposed to be absolutely continuous according to the definition of Vitali, and such that along them the integral which it is wished to study exists and is finite. Furthermore, and this is indeed the most important point to set in evidence, the existence theorems are given almost all for the classes of curves which I have named complete. In the parametric case a complete class is simply a totality of rectifiable curves to which belong all those curves which are at the same time limiting curves of the class and rectifiable. For the ordinary non-parametric case one substitutes for rectifiability in this definition the two properties which I have indicated just above. The theorems of existence assume in this way the maximum of generality; they are completely independent of the use of extremal curves; and they have a form applicable also to problems of extrema which could not be approached in any way by already known methods.

It is important to notice that the method of demonstrating the existence of extrema by the use of semi-continuity permits further the consideration of classes of integrals which are not everywhere semi-continuous. Of this I have given examples in my book, studying those types of integrals which have been the subject of noteworthy researches by C. Carathéodory. Such integrals have in general extremizing curves with corner points. For an integral of this type I have given a demonstration of the existence of an extremum by reducing the problem to one with an integral which possesses everywhere the property of semi-continuity 
and which is intimately related to the integral originally given. The same procedure permits me then to treat completely also the classical problem of Newton concerning the solid of revolution of minimum resistance, a problem whose solution, indicated by Newton himself, was completely justified only in 1900 by A. Kneser.

5. Extremaloids and Extremals. When the existence of an extremum has been proved it becomes necessary to pass to the discussion of the analytic properties of the extremizing curves. It is evident that these properties are intimately related to the character of the class of curves in which the extremum has been sought; and for the study of them the generality of the class considered must necessarily be restricted.

Limiting myself to the classes customarily considered in the calculus of variations, and moreover maintaining with respect to the character of the curves themselves all of the generality which I have indicated above, I am led to introduce the concept of an extremaloid which is a generalization of the known concept extremal.

In the parametric form, denoting by $F\left(x, y, x^{\prime}, y^{\prime}\right)$ the integrand function, I designate as an extremaloid every rectifiable curve satisfying the two equations

$\int_{0}^{s} F_{x} d s-\frac{d}{d s} \int_{0}^{s} F_{x^{\prime}} d s=c_{1}, \quad \int_{0}^{s} F_{y} d s-\frac{d}{d s} \int_{0}^{s} F_{y^{\prime}} d s=c_{2}$,

where $s$ represents the length of arc. These equations are evidently generalizations of those of the extremals; they have the advantage of being in a form which permits them to be satisfied also by a curve which is only rectifiable. Generalizing a classical argument I prove that every extremizing curve, relative to all those of a given neighborhood of it joining the same two end points, is necessarily an extremaloid if it is interior to the field considered. I obtain thus the extension of Euler's condition to curves which are rectifiable only. I prove next that, for the quasiregular normal integrals, the extremaloids are necessarily 
extremals, and I have here the means of treating an important case in which the extremals present singularities arising from the vanishing of the coefficient of the term of the second order in their equation. For the case of the quasi-regular normal integrals again, I prove that for fields of a very general type, but with boundaries having no corner points pointing toward the interior of the field itself, all the extremizing curves have everywhere continously turning tangents even on the parts which touch the boundary. For special domains, or for unlimited domains when the behavior of the function $F$ as the point $(x, y)$ tends to infinity is suitably restricted, the extremizing curve turns out to be simply an extremal. From this $I$ derive theorems of existence for extremals joining two given points not necessarily near to each other, and for periodic extremals.

I study further the uniqueness of the extremizing curves and of the extremal joining two given points.

It goes without saying that the known conditions for an extremum are re-deduced, those of Weierstrass and Legendre being, as has been already stated, an immediate consequence of the semi-continuity which must be verified on every extremizing curve.

I find moreover the known sufficient conditions for a relative extremum. Here I solve the problem first for a small region, reducing the study of the given integral to that of another integral possessing everywhere semicontinuity, and applying the results already obtained. The same method makes it possible to solve the analogous problem in the general case, which can also be treated by applying the results obtained for a small region.

For the non-parametric integrals greater difficulties in the case of Euler's condition present themselves, depending upon the fact that one can not be sure a priori of the existence of the integrals which occur in the equations of the extremaloids and whose integrands are the derivatives with respect to $y$ and $y^{\prime}$ of the function $f\left(x, y, y^{\prime}\right)$ taken 
[March-April,

at the points of the extremizing arc. The existence of these integrals seems probable, but I have not succeeded so far either in proving it for all cases or in constructing an example for which they do not exist. Nevertheless I have given in this connection propositions of a very general character. For example I have shown that every extremizing curve, relative to all those in a neighborhood of it joining two given points, is an extremaloid provided that it is interior to the field under consideration and has bounded difference quotients; and I have also shown that the condition relative to the difference quotients can be altogether suppressed for a noteworthy and extensive category of integrals which includes precisely those integrals which for the most part present themselves in the applications of the calculus of variations. For this same category of integrals and also for another still more general, I have moreover proved that every extremaloid is necessarily an extremal. I have studied especially regular integrals, and those which are quasi-regular normal, seeking to attain results analogous to those for the parametric case. Here the extremizing curves may have first derivatives of their ordinates which become infinite; but I have given conditions sufficient to insure that this shall not occur.

All the arguments made for the parametric case are taken up again for the integrals in non-parametric form; and by the method already indicated one finds here also the known necessary and the known sufficient conditions for a relative extremum. The problem which I have discussed hitherto is that of a non-isoperimetric extremum; but the method described is applied in the second volume of my Fondamenti to isoperimetric problems also.

The method of procedure, as is evident from what has been said, does not depend in any way upon the theory of differential equations; indeed it adds to that theory a contribution in the domain of the theorems of existence.

Bologna, Italy 